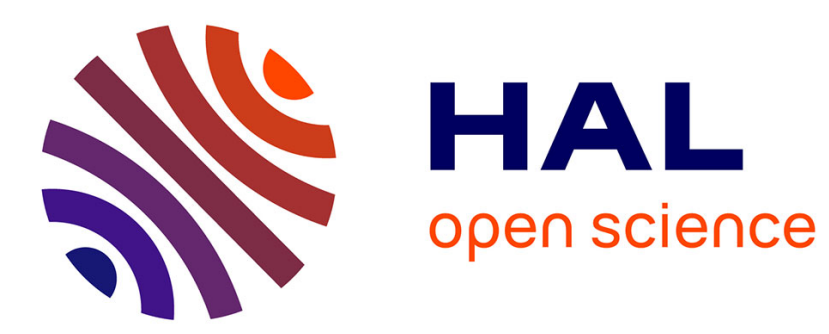

\title{
Interspecific density-dependent model of predator-prey relationship in the chemostat
}

\author{
Tahani Mtar, Radhouane Fekih-Salem, Tewfik Sari
}

\section{To cite this version:}

Tahani Mtar, Radhouane Fekih-Salem, Tewfik Sari. Interspecific density-dependent model of predator-prey relationship in the chemostat. International Journal of Biomathematics, 2021, 14 (1), pp.2050086. 10.1142/S1793524520500862 . hal-02445761v2

\section{HAL Id: hal-02445761 \\ https://hal.science/hal-02445761v2}

Submitted on 10 Sep 2020

HAL is a multi-disciplinary open access archive for the deposit and dissemination of scientific research documents, whether they are published or not. The documents may come from teaching and research institutions in France or abroad, or from public or private research centers.
L'archive ouverte pluridisciplinaire HAL, est destinée au dépôt et à la diffusion de documents scientifiques de niveau recherche, publiés ou non, émanant des établissements d'enseignement et de recherche français ou étrangers, des laboratoires publics ou privés. 


\title{
INTERSPECIFIC DENSITY-DEPENDENT MODEL OF PREDATOR-PREY RELATIONSHIP IN THE CHEMOSTAT
}

\author{
Tahani MTAR
}

University of Tunis El Manar, National Engineering School of Tunis, LAMSIN, Tunis, Tunisia thani.mtar@gmail.com

Radhouane FEKIH-SALEM

University of Tunis El Manar, National Engineering School of Tunis, LAMSIN, Tunis, Tunisia University of Monastir, Higher Institute of Computer Science of Mahdia, 5111, Mahdia, Tunisia radhouene.fekihsalem@isima.rnu.tn

Tewfik SARI

ITAP, Univ Montpellier, INRAE, Institut Agro, Montpellier, France tewfik.sari@inrae.fr

Received (20 Jan. 2020)

Revised (8 Sep. 2020)

\begin{abstract}
The objective of this study is to analyze a model of competition for one resource in the chemostat with general inter-specific density-dependent growth rates, taking into account the predator-prey relationship. This relationship is characterized by the fact that the prey species promotes the growth of the predator species which in turn inhibits the growth of the first species. The model is a three-dimensional system of ordinary differential equations. With the same dilution rates, the model can be reduced to a planar system where the two models have the same local and even global behavior. The existence and stability conditions of all steady states of the reduced model in the plane are determined according to the operating parameters. Using the nullcline method, we present a geometric characterization of the existence and stability of all equilibria showing the multiplicity of coexistence steady states. The bifurcation diagrams illustrate that the steady states can appear or disappear only through saddle-node or transcritical bifurcations. Moreover, the operating diagrams describe the asymptotic behavior of this system by varying the control parameters and show the effect of the inhibition of predation on the emergence of the bistability region and the reduction until the disappearance of the coexistence region by increasing this inhibition parameter.
\end{abstract}

Keywords: Bifurcation; chemostat; coexistence; predator-prey relationship; operating diagram.

\section{Introduction}

The culture of microorganisms occurs in a laboratory apparatus called "chemostat", which was invented in 1950. It is often used in various scientific fields such as ecology, biology, genetics, etc. It is also used as a model of the wastewater treatment 
process. We recall the classical chemostat model when two species $x_{1}$ and $x_{2}$ are in competition for a single substrate $S$ :

$$
\left\{\begin{array}{l}
\dot{S}=D\left(S_{i n}-S\right)-\frac{1}{\gamma_{1}} f_{1}(S) x_{1}-\frac{1}{\gamma_{2}} f_{2}(S) x_{2} \\
\dot{x}_{1}=\left(f_{1}(S)-D\right) x_{1} \\
\dot{x}_{2}=\left(f_{2}(S)-D\right) x_{2}
\end{array}\right.
$$

where $S(t)$ is the concentration of the substrate at time $t ; x_{1}(t)$ and $x_{2}(t)$ the concentrations of the species at time $t ; D$ and $S_{i n}$ are, respectively, the dilution rate and the concentration of the substrate in the feed device; $f_{1}(S)$ and $f_{2}(S)$ are the growth functions and $\gamma_{1}, \gamma_{2}$ the yield constants, which can be easily normalized to 1 , using the change of variable $x_{i} / \gamma_{i} \rightarrow x_{i}, i=1,2$. Since at steady state, we cannot have, in general, $f_{1}(S)=f_{2}(S)=D$, the model (1.1) predicts that at most one species can survive the competition, the one with the lowest break-even concentration. This classical result is called the Competitive Exclusion Principle (CEP) and which has been corroborated by the experiences of Hansen and Hubbell [15]. But, the CEP contradicts the biodiversity that is observed in microbial ecosystems as well as in waste-water treatment processes and bioreactors [23, 24]. Thus, the classical chemostat model is unable to reproduce reality even qualitatively and new hypotheses should be considered in order to reconcile the theory and the experimental results.

Several mechanisms of coexistence in the chemostat were considered in the literature, such as the intra- and interspecific competition between the species $[1,3,5,13,27]$, the flocculation of the species $[9,11,12,14]$, and the densitydependence of the growth functions $[6-8,10,21]$. Mathematical study of several chemostat-like models can be found in the monograph [25], see also [16].

On the other hand, some studies have shown that species can coexist considering predator-prey interactions in the chemostat, [2, 20, 22]. For instance, in [2, 20], a predator-prey model in the chemostat has been analyzed with Holling's type- $I I$ functional responses and distinct removal rates, where the model presents a rich set of behaviors with coexistence, transcritical and Hopf bifurcations with occurrence of stable limit cycles. Li and Kuang [20] show that the behavior of the system can be affected according to the death rate of species where the system can present oscillatory solutions. In [22], authors have considered the case of more complex food webs where two preys consume a single nutrient and the predator consumes only the preys. With the general monotone response functions and the same dilution rates, it was shown that the coexistence steady state is stable as long as it exists.

In this context, we will consider an interspecific density-dependent model of two species competing for a single nutrient in the chemostat where the removal rates of the substrate and the biomass are equal. This model introduced by El Hajji et al. 
[7] is written as follows:

$$
\left\{\begin{array}{l}
\dot{S}=D\left(S_{i n}-S\right)-f_{1}\left(S, x_{2}\right) x_{1}-f_{2}\left(S, x_{1}\right) x_{2}, \\
\dot{x}_{1}=\left(f_{1}\left(S, x_{2}\right)-D\right) x_{1}, \\
\dot{x}_{2}=\left(f_{2}\left(S, x_{1}\right)-D\right) x_{2},
\end{array}\right.
$$

where $x_{1}(t)$ and $x_{2}(t)$ are, respectively, the concentrations of prey and predator at time $t$. We assume that $f_{1}$ depends only on $S$ and $x_{2}$, and $f_{2}$ depends only on $S$ and $x_{1}$, and are increasing in the variable $S$. To demonstrate how interspecific interference can explain the coexistence of competing species in chemostat, El Hajji [6], considered three types of interspecific interactions: inhibition, mutualism and food web (or predator-prey relationship). The inhibition is characterized by the fact that each species inhibits the growth of the other species. The mutualism is characterized by the fact that each species promotes the growth of the other species. The predator-prey relationship is characterized by the fact that one species (the prey) promotes the growth of the other species (the predator) which in turn inhibits the growth of the prey species.

El Hajji and Rapaport [8] have considered the first type where $f_{1}$ is decreasing in $x_{2}$ and $f_{2}$ is decreasing in $x_{1}$. In $[6,8]$, it was proved that the coexistence of two species is impossible which confirms the CEP. Fekih-Salem et al. [10] have provided an extension of the results in [8] to general intra- and interspecific density-dependent growth rates $f_{i}$ which depends on both species $x_{1}$ and $x_{2}$, not only on $x_{j}$ where $j \neq i$, and distinct removal rates.

The second type is the obligate mutualistic relationship where the function $f_{i}$ is increasing in the variable $x_{j}$. Using general monotonic functional responses, the model exhibits the coexistence where the positive steady state is not necessarily unique [7].

Here, we will give a particular attention to the third type of interaction which describes the predator-prey relationship where the function $f_{1}$ is decreasing in the variable $x_{2}$ while the function $f_{2}$ is increasing in the variable $x_{1}$. In this case, El Hajji [6] has shown that system (1.2) may exhibit the coexistence and the bistability with a multiplicity of positive steady states.

Our main objective in this work is to give a quite comprehensive analysis of model (1.2) in the case of the predator-prey relationship. The existence and stability conditions of all steady states are provided according to the control parameters $S_{i n}$ and $D$. Using the nullcline method, we present a geometric characterization that describes all steady states of the model and determines the nature of bifurcation and the emergence of coexistence steady states. Varying the operating parameter $S_{i n}$, the bifurcation diagrams show all steady states that can appear or disappear only through saddle-node or transcritical bifurcations. All these results have not been done in [6]. Last but not least, we analyze the operating diagram to determine the behavior of system with respect to the parameters $S_{i n}$ and $D$, and we study the effect of the inhibition of predation on the behavior of system in this operating plane $\left(S_{i n}, D\right)$. These questions were not considered in [6]. 
This paper is organized as follows. In the next section, we first introduce the general assumptions on the growth functions and we provide a preliminary result on positivity and boundedness of solutions. Then, we define the reduced model in the plane from the three-dimensional system (1.2). In Section 3, we analyze the reduced model by determining the conditions of existence and stability of steady states Then, we give the result of global stability of the three-dimensional system (1.2). In Section 4, the operating diagrams describe the existence and the stability of each steady state according to control parameters $D$ and $S_{i n}$. Considering specific growth rates, numerical simulations are presented in Section 5. The conclusion is given in the last Section 6. All proofs of propositions and lemmas are reported in Appendix A. With specific growth rates satisfying the general assumptions, the number of positive extrema of some functions is given in Appendix B. All the parameter values used in simulations are provided in Appendix C.

\section{Assumptions and reduced model}

In what follows, we consider model (1.2). For $i=1,2, j=1,2, i \neq j$, we first make the following general assumptions on the growth rates $f_{i}$.

(H0) $f_{i}: \mathbb{R}_{+}^{2} \longrightarrow \mathbb{R}_{+}$is continuously differentiable.

(H1) $f_{i}\left(0, x_{j}\right)=0$, for all $x_{j} \geq 0$.

(H2) $\frac{\partial f_{i}}{\partial S}\left(S, x_{j}\right)>0$, for all $S \geq 0, x_{1}>0$ and $x_{2} \geq 0$.

(H3) $\frac{\partial f_{1}}{\partial x_{2}}\left(S, x_{2}\right)<0$ and $\frac{\partial f_{2}}{\partial x_{1}}\left(S, x_{1}\right)>0$, for all $S>0, x_{1} \geq 0$ and $x_{2} \geq 0$.

(H4) $f_{2}(S, 0)=0$, for all $S>0$.

Assumption (H1) means that the substrate is necessary for the growth of the two species. Assumption (H2) means that the growth rate of each species increases with the concentration of the substrate. Assumption (H3) means that the growth of the first species $x_{1}$ is inhibited by the second species $x_{2}$, while the growth of second species $x_{2}$ increases with the presence of first species $x_{1}$. Assumption (H4) means that the prey species $x_{1}$ is necessary for the growth of the predator species $x_{2}$. These assumptions are satisfied by the following growth rates:

$$
f_{1}\left(S, x_{2}\right)=\frac{m_{1} S}{K_{1}+S} \frac{1}{1+x_{2} / L_{1}}, \quad f_{2}\left(S, x_{1}\right)=\frac{m_{2} S}{K_{2}+S} \frac{x_{1}}{L_{2}+x_{1}},
$$

where $m_{1}, m_{2}$ denote the maximum growth rates; $K_{1}, K_{2}$ and $L_{2}$ denote the Michaelis-Menten constants; $L_{1}$ represents the inhibition factor due to $x_{2}$ for the growth of the species $x_{1}$. In order to show that our model (1.2) preserves the biological meaning, we will show the following property.

Proposition 2.1. Assume that Hypotheses (HO), (H1) and (H4) hold. For any nonnegative initial condition, the solution of system (1.2) exists for all $t \geq 0$, re- 
mains nonnegative and is bounded. In addition, the set

$$
\Omega=\left\{\left(S, x_{1}, x_{2}\right) \in \mathbb{R}_{+}^{3}: S+x_{1}+x_{2}=S_{i n}\right\},
$$

is positively invariant and is a global attractor for the dynamics (1.2).

In what follows, it is convenient to use the abbreviation LES for Locally Exponentially Stable steady state and GAS for Globally Asymptotically Stable steady state, in the positive cone. In the following, we prove that the three-dimensional system (1.2) can be reduced to a two-dimensional system. Let $z=S+x_{1}+x_{2}$ be the total mass density. Writing in the variables $\left(z, x_{1}, x_{2}\right)$, system (1.2) becomes

$$
\left\{\begin{array}{l}
\dot{z}=D\left(S_{i n}-z\right), \\
\dot{x}_{1}=\left(f_{1}\left(z-x_{1}-x_{2}, x_{2}\right)-D\right) x_{1}, \\
\dot{x}_{2}=\left(f_{2}\left(z-x_{1}-x_{2}, x_{1}\right)-D\right) x_{2} .
\end{array}\right.
$$

Let $\left(z(t), x_{1}(t), x_{2}(t)\right)$ be a solution of (2.2). From the first equation of (2.2), we deduce that $z(t)=S_{i n}+\left(z(0)-S_{i n}\right) e^{-D t}$. Thus, $\left(x_{1}(t), x_{2}(t)\right)$ is a solution of the following asymptotically autonomous system:

$$
\left\{\begin{array}{l}
\dot{x}_{1}=\left(f_{1}\left(S_{i n}+\left(z(0)-S_{i n}\right) e^{-D t}-x_{1}-x_{2}, x_{2}\right)-D\right) x_{1} \\
\dot{x}_{2}=\left(f_{2}\left(S_{i n}+\left(z(0)-S_{i n}\right) e^{-D t}-x_{1}-x_{2}, x_{1}\right)-D\right) x_{2} .
\end{array}\right.
$$

Then, system (2.3) converges toward the following autonomous system:

$$
\left\{\begin{array}{l}
\dot{x}_{1}=\left(f_{1}\left(S_{i n}-x_{1}-x_{2}, x_{2}\right)-D\right) x_{1}, \\
\dot{x}_{2}=\left(f_{2}\left(S_{i n}-x_{1}-x_{2}, x_{1}\right)-D\right) x_{2} .
\end{array}\right.
$$

This is called the reduced model since it is simply the restriction of (2.3) to the invariant plane defined by $z(t)=S_{i n}$, for all $t \geq 0$. Using Thieme's result [26], one can prove that the asymptotic behavior of the three-dimensional system (1.2) can be deduced from the asymptotic behavior of the two-dimensional system (2.4).

In the following, we focus on the study of the reduced system (2.4). This system is defined on the set

$$
\mathcal{M}:=\left\{\left(x_{1}, x_{2}\right) \in \mathbb{R}_{+}^{2}: x_{1}+x_{2} \leq S_{i n}\right\},
$$

which is positively invariant for system (2.4). Indeed, for $i=1,2$, if $x_{i}=0$ then $\dot{x}_{i}=0$. As a result, the axis $x_{i}=0$ is invariant under system (2.4) because the function

$$
t \longmapsto\left(0, x_{2}(t)\right), \quad\left(\text { resp. } t \longmapsto\left(x_{1}(t), 0\right)\right),
$$

is a solution of (2.4). By uniqueness of solutions, the axis $x_{i}=0$ cannot be reached in finite time by trajectories for $x_{i}(0)>0$. Furthermore, we have

$$
\left(x_{1}+x_{2}\right)\left(t_{1}\right)=S_{i n}, \quad \text { for some } \quad t_{1} \geq 0 \quad \Longrightarrow \quad \dot{x}_{i}\left(t_{1}\right)=-D x_{i}\left(t_{1}\right) \leq 0 .
$$




\section{Analysis of the reduced and the three-dimensional models}

The steady states of (2.4) are given by the solutions of the following system:

$$
\left\{\begin{array}{l}
0=\left(f_{1}\left(S_{i n}-x_{1}-x_{2}, x_{2}\right)-D\right) x_{1}, \\
0=\left(f_{2}\left(S_{i n}-x_{1}-x_{2}, x_{1}\right)-D\right) x_{2} .
\end{array}\right.
$$

From (H4), we deduce that system (2.4) cannot have a steady state where the first population is extinct while the second species survives. Otherwise, if $x_{1}=0$ one has $x_{2}=0$, then the steady state $E_{0}=(0,0)$, called the washout, always exists.

Assume that Hypotheses (H0) to (H2) hold. For all $0 \leqslant D<f_{1}(+\infty, 0)$, we define the usual break-even concentration by

$$
S=\lambda(D),
$$

which is the unique solution of equation $f_{1}(S, 0)=D$. If $D \geq f_{1}(+\infty, 0)$, we put $\lambda(D)=+\infty$. The following result determines the existence of the steady state corresponding to the extinction of the second population $x_{2}$ :

Proposition 3.1. Assume that assumptions (HO)-(H2) hold. System (2.4) has the steady state $E_{1}=\left(\tilde{x}_{1}, 0\right)$ where $\tilde{x}_{1}=S_{\text {in }}-\lambda(D)$. It exists if and only if,

$$
S_{\text {in }}>\lambda(D) \text {. }
$$

In the following, we determine the existence condition of the positive steady state $E^{*}=\left(x_{1}^{*}, x_{2}^{*}\right)$ of system (2.4), where both populations survive. The components $x_{1}=x_{1}^{*}$ and $x_{2}=x_{2}^{*}$ of $E^{*}$ must be the solutions of (3.1) with $x_{1}>0$ and $x_{2}>0$. Therefore, $x_{1}^{*}$ and $x_{2}^{*}$ are the solutions of the set of the following equations

$$
\left\{\begin{array}{l}
f_{1}\left(S_{i n}-x_{1}-x_{2}, x_{2}\right)=D \\
f_{2}\left(S_{i n}-x_{1}-x_{2}, x_{1}\right)=D .
\end{array}\right.
$$

That is to say, (3.4) must have a positive solution $\left(x_{1}^{*}, x_{2}^{*}\right)$ in

$$
M^{o}:=\left\{\left(x_{1}, x_{2}\right) \in\left(\mathbb{R}_{+}^{*}\right)^{2}: x_{1}+x_{2}<S_{i n}\right\},
$$

which is the interior of $\mathcal{M}$. To solve (3.4) in $M^{o}$, we state the following results:

Lemma 3.2. Assume that hypotheses (HO) to (H3) and condition (3.3) hold. The equation $f_{1}\left(S_{i n}-x_{1}-x_{2}, x_{2}\right)=D$ defines a smooth decreasing function

$$
\begin{aligned}
F_{1}:\left[0, \tilde{x}_{1}\right] & \longrightarrow\left[0, \tilde{x}_{2}\right] \\
x_{1} & \longmapsto F_{1}\left(x_{1}\right)=x_{2},
\end{aligned}
$$

such that $F_{1}\left(\tilde{x}_{1}\right)=0, F_{1}(0)=\tilde{x}_{2}$ and $-1<F_{1}^{\prime}\left(x_{1}\right)<0$ for all $x_{1} \in\left[0, \tilde{x}_{1}\right]$, where $x_{2}=F_{1}\left(x_{1}\right)$ is the unique solution of the equation $f_{1}\left(S_{i n}-x_{1}-x_{2}, x_{2}\right)=D$ and $\tilde{x}_{2}$ is the unique solution of the equation $f_{1}\left(S_{i n}-x_{2}, x_{2}\right)=D$. Furthermore, the graph $\gamma_{1}$ of $F_{1}$ lies in $M^{o}$ (see Fig. $\left.1(b)\right)$. More precisely, $\left(x_{1}, F_{1}\left(x_{1}\right)\right) \in M^{o}$ for all $\left.x_{1} \in\right] 0, \tilde{x}_{1}[$.

To determine the function defined by the equation $f_{2}\left(S_{i n}-x_{1}-x_{2}, x_{1}\right)=D$, we will have need to define the solutions of the equation $f_{2}\left(S_{i n}-x_{1}, x_{1}\right)=D$. 
Lemma 3.3. Assume that assumptions (HO) to (H4) hold. The equation

$$
f_{2}\left(S_{i n}-x_{1}, x_{1}\right)=D
$$

has a solution in $\left[0, S_{i n}\right]$ if and only if,

$$
\max _{x_{1} \in\left[0, S_{i n}\right]} f_{2}\left(S_{i n}-x_{1}, x_{1}\right) \geq D .
$$

Generically, we have an even number of solutions in $\left[0, S_{i n}\right]$.

(a)

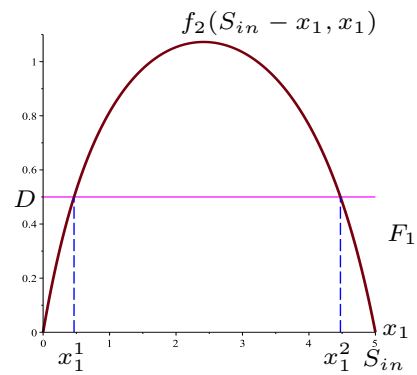

$(b)$

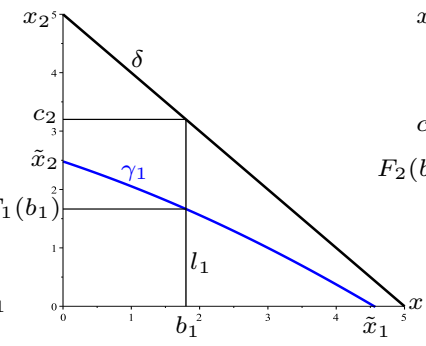

(c)

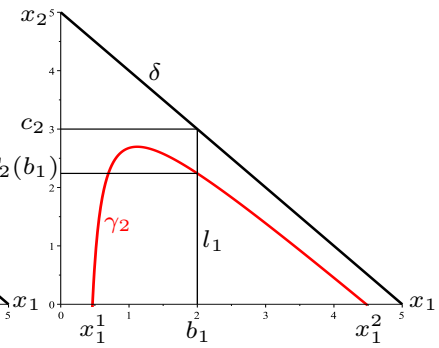

Fig. 1. When the specific growth functions are given by (2.1): (a) number of solutions of equation (3.5), (b) definition of $F_{1},(c)$ definition of $F_{2}$ where $\delta$ is the line defined by $x_{1}+x_{2}=S_{i n}$.

For simplicity, we add the following assumption which is satisfied by the specific growth rates (2.1).

(H5) Equation (3.5) has at most two solutions $x_{1}^{1}$ and $x_{1}^{2}$ in $\left[0, S_{i n}\right]$ (see Fig. 1(a)).

With the specific growth rates (2.1), this property is satisfied (see Appendix B). The general case where the function $x_{1} \mapsto f_{2}\left(S_{i n}-x_{1}, x_{1}\right)$ is multimodal can be treated similarly, without added difficulty. In this particular case, we obtain the following result:

Lemma 3.4. Assume that hypotheses (HO) to (H5) and condition (3.6) hold. The equation $f_{2}\left(S_{\text {in }}-x_{1}-x_{2}, x_{1}\right)=D$ defines a function

$$
\begin{aligned}
F_{2}:\left[x_{1}^{1}, x_{1}^{2}\right] & \longrightarrow\left[0, S_{i n}[\right. \\
x_{1} & \longmapsto F_{2}\left(x_{1}\right)=x_{2},
\end{aligned}
$$

such that $F_{2}\left(x_{1}^{1}\right)=F_{2}\left(x_{1}^{2}\right)=0$ and $-1<F_{2}^{\prime}\left(x_{1}\right)$ for all $x_{1} \in\left[x_{1}^{1}, x_{1}^{2}\right]$, where $x_{2}=F_{2}\left(x_{1}\right)$ is the unique solution of the equation $f_{2}\left(S_{i n}-x_{1}-x_{2}, x_{1}\right)=D$, and $x_{1}^{1}$ and $x_{1}^{2}$ are the solutions of (3.5). Furthermore, the graph $\gamma_{2}$ of $F_{2}$ lies in $M^{o}$ (see Fig. 1(c)). More precisely, $\left(x_{1}, F_{2}\left(x_{1}\right)\right) \in M^{o}$ for all $\left.x_{1} \in\right] x_{1}^{1}, x_{1}^{2}[$.

Note that the function $F_{2}$ has a unique extremum in $\left[x_{1}^{1}, x_{1}^{2}\right]$ with the specific growth functions (2.1) (see Fig. 1(c) and Appendix B). One can see that $\tilde{x}_{1}, x_{1}^{1}$ and $x_{1}^{2}$ are simply the solutions of the following equations

$$
f_{1}\left(S_{\text {in }}-\tilde{x}_{1}, 0\right)=D, \quad f_{2}\left(S_{\text {in }}-x_{1}^{1}, x_{1}^{1}\right)=D, \quad f_{2}\left(S_{\text {in }}-x_{1}^{2}, x_{1}^{2}\right)=D .
$$


These quantities represent the coordinates of the intersections of the curves $\gamma_{1}$ and $\gamma_{2}$ with the $x_{2}=0$ axis. Their relative positions play a major role in the behavior of system (2.4). In fact, there exists three cases that must be distinguished:

Case 1: $\tilde{x}_{1}<x_{1}^{1}<x_{1}^{2}$, Case $2: x_{1}^{1}<\tilde{x}_{1}<x_{1}^{2}$, Case $3: x_{1}^{1}<x_{1}^{2}<\tilde{x}_{1}$.

When hypotheses (H0) to (H5) and condition (3.6) hold, we have the following equivalences (see Fig. 1(a)):

$$
\begin{array}{lll}
f_{2}\left(\lambda(D), S_{i n}-\lambda(D)\right)<D & \Leftrightarrow & \text { Case } 1 \text { or Case } 3 \\
f_{2}\left(\lambda(D), S_{i n}-\lambda(D)\right)>D & \Leftrightarrow & \text { Case } 2 .
\end{array}
$$

As a consequence of the previous lemmas, we obtain the following result which gives the condition of existence of positive steady states.

Proposition 3.5. Assume that hypotheses (HO) to (H5), and conditions (3.3) and (3.6) hold. A positive steady state $E^{*}=\left(x_{1}^{*}, x_{2}^{*}\right)$ of (2.4) exists if and only if the curves $\gamma_{1}$ and $\gamma_{2}$ have a positive intersection, that is, $\left(x_{1}^{*}, x_{2}^{*}\right)$ is a positive solution of equations

$$
x_{2}=F_{1}\left(x_{1}\right) \quad \text { and } \quad x_{2}=F_{2}\left(x_{1}\right) .
$$

The following proposition provides the number of positive steady states of (2.4).

Proposition 3.6. Assume that hypotheses (HO) to (H5), and conditions (3.3) and (3.6) hold.

(1) When Case 1 holds, then there is no positive steady state.

(2) When Case 2 holds, then there exists at least one positive steady state. Generically, the system has an odd number of positive steady states.

(3) When Case 3 holds, then generically system (2.4) has no positive steady state or an even number of positive steady states.
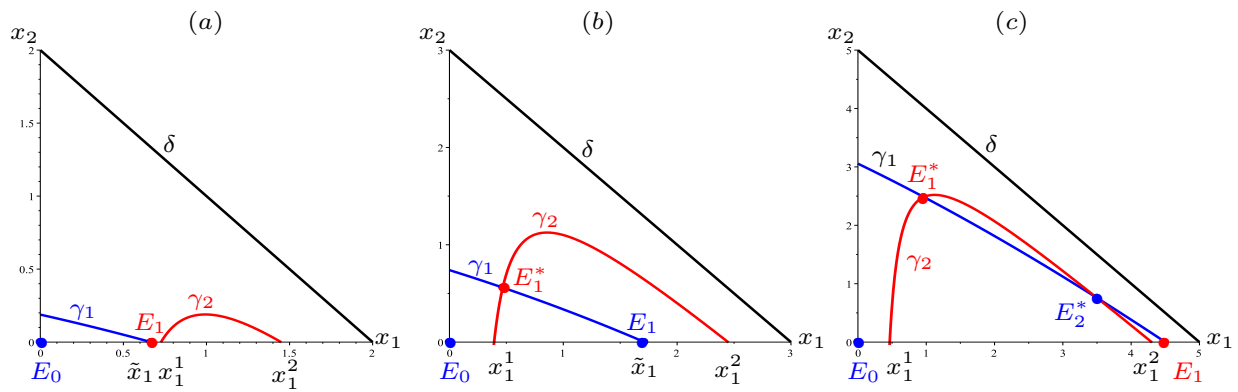

Fig. 2. Number of positive steady states:(a) Case 1: no intersection, (b) Case 2: an odd number of intersections, (c) Case 3: an even number of intersections. In all figures, we have chosen the red color for LES steady state and the blue color for unstable steady state.

The following result provides the local stability condition of each steady state of system (2.4). 
Proposition 3.7. Assume that hypotheses (HO) to (H5) hold, we have

(1) $E_{0}=(0,0)$ is LES if and only if $S_{\text {in }}<\lambda(D)$.

(2) $E_{1}=\left(\tilde{x}_{1}, 0\right)$ is LES if and only if $f_{2}\left(\lambda(D), S_{i n}-\lambda(D)\right)<D$.

(3) $E^{*}=\left(x_{1}^{*}, x_{2}^{*}\right)$ is LES if and only if $F_{1}^{\prime}\left(x_{1}^{*}\right)<F_{2}^{\prime}\left(x_{1}^{*}\right)$.

The next proposition determines the conditions of global asymptotic stability of each steady state of (2.4).

Proposition 3.8. Assume that hypotheses (HO) to (H5) hold.

(1) $E^{*}$ is GAS in $M^{o}$ if and only if $f_{2}\left(\lambda(D), S_{\text {in }}-\lambda(D)\right)>D$ and (3.9) has a unique solution.

(2) $E_{1}$ is $G A S$ in $M^{o}$ if and only if $f_{2}\left(\lambda(D), S_{i n}-\lambda(D)\right) \leq D$ and (3.9) has no solution.

(3) $E_{0}$ is GAS in $M^{o}$ if and only if $S_{i n} \leq \lambda(D)$.

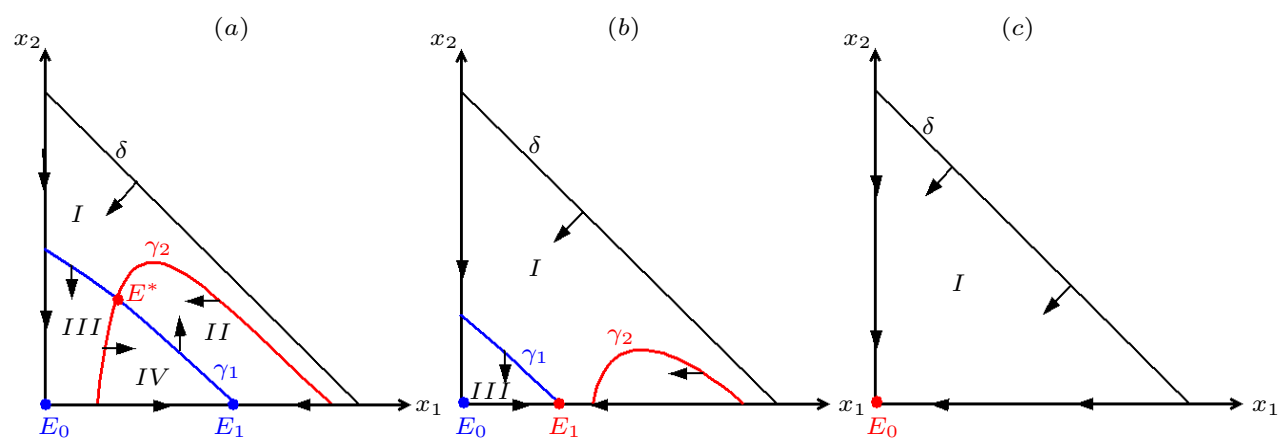

Fig. 3. Global stability of steady states of (2.4):(a) Item 1 of Proposition 3.8, where $E^{*}$ is GAS, (b) Item 2 of Proposition 3.8, where Case 1 holds and $E_{1}$ is GAS, (c) Item 3 of Proposition 3.8, where $E_{0}$ is GAS.

In what follows, we will determine the global asymptotic stability of each steady state of three-dimensional system (1.2), which is deduced from that of reduced model (2.4). Notice that to the steady states of (1.2)

$$
\mathcal{E}_{0}=\left(S_{i n}, 0,0\right), \quad \mathcal{E}_{1}=\left(S_{\text {in }}-\tilde{x}_{1}, \tilde{x}_{1}, 0\right), \quad \mathcal{E}^{*}=\left(S_{\text {in }}-x_{1}^{*}-x_{2}^{*}, x_{1}^{*}, x_{2}^{*}\right)
$$

correspond the steady states of (2.4), respectively,

$$
E_{0}=(0,0), \quad E_{1}=\left(\tilde{x}_{1}, 0\right), \quad E^{*}=\left(x_{1}^{*}, x_{2}^{*}\right) .
$$

Using the convergence theorem [25] (or Thieme's result [26]), we deduce that the asymptotic behavior of the solution of three order system (1.2) is the same as the asymptotic behavior of reduced system (2.4). Now, the main result can be stated.

Theorem 3.9. Assume that hypotheses (HO) to (H5) hold.

(1) $\mathcal{E}^{*}$ is GAS in the interior of $\Omega$ if and only if $f_{2}\left(\lambda(D), S_{i n}-\lambda(D)\right)>D$ and (3.9) has a unique solution. 
(2) $\mathcal{E}_{1}$ is GAS in the interior of $\Omega$ if and only if $f_{2}\left(\lambda(D), S_{i n}-\lambda(D)\right) \leq D$ and (3.9) has no solution.

(3) $\mathcal{E}_{0}$ is $G A S$ in $\Omega$ if and only if $S_{\text {in }} \leq \lambda(D)$.

The necessary and sufficient conditions of existence, local and global stability of all steady states of (1.2) are summarized in Table 1.

Table 1. Necessary and sufficient conditions of existence and stability of steady states of system (1.2).

\begin{tabular}{llll}
\hline & Existence & Local stability & Global stability \\
\hline $\mathcal{E}_{0}$ & always exists & $S_{i n}<\lambda(D)$ & $S_{i n} \leq \lambda(D)$ \\
$\mathcal{E}_{1}$ & $S_{i n}>\lambda(D)$ & $f_{2}\left(\lambda(D), S_{i n}-\lambda(D)\right)<D$ & $f_{2}\left(\lambda(D), S_{i n}-\lambda(D)\right) \leq D$, \\
& & $(3.9)$ has no solution \\
$\mathcal{E}^{*}$ & $(3.9)$ has a solution & $F_{2}^{\prime}\left(x_{1}^{*}\right)>F_{1}^{\prime}\left(x_{1}^{*}\right)$ & $f_{2}\left(\lambda(D), S_{i n}-\lambda(D)\right)>D$, \\
& & $(3.9)$ has a unique solution \\
\hline
\end{tabular}

\section{Operating diagram}

The operating diagram shows how the system behaves when we vary the two control parameters $S_{\text {in }}$ and $D$. It will be used to describe the behavior of system (1.2) in different regions of the operating plan. All other biological parameters are those of the growth functions and are fixed since they depend on the nature of the organisms and the substrate introduced into the bioreactor. All the biological parameter values used are provided in Table 3 by considering the specific growth functions (2.1). Note that the operating diagrams of the interspecific density-dependent model (1.2) have not been studied in the existing literature. Let $\Upsilon_{1}$ be the curve of equation $D=f_{1}\left(S_{\text {in }}, 0\right)$,

$$
\Upsilon_{1}:=\left\{\left(S_{i n}, D\right): D=f_{1}\left(S_{i n}, 0\right)\right\} .
$$

Recall that $\tilde{x}_{1}=S_{i n}-\lambda(D)$ and $x_{1}^{i}, i=1,2$ are the solutions of equation (3.5) defined by $f_{2}\left(S_{i n}-x_{1}, x_{1}\right)=D$. Therefore, $\tilde{x}_{1}$ and $x_{1}^{i}$ depend on the operating parameters $S_{i n}$ and $D$. We denote them by $\tilde{x}_{1}\left(S_{i n}, D\right)$ and $x_{1}^{i}\left(S_{i n}, D\right), i=1,2$. Define the curve $\Upsilon_{2}$ of equation $\tilde{x}_{1}\left(S_{i n}, D\right)=x_{1}^{2}\left(S_{i n}, D\right)$ by the set

$$
\Upsilon_{2}:=\left\{\left(S_{i n}, D\right): \tilde{x}_{1}\left(S_{i n}, D\right)=x_{1}^{2}\left(S_{i n}, D\right)\right\} .
$$

Let $\Upsilon_{3}$ be the curve in the plan $\left(S_{i n}, D\right)$ which corresponds to a saddle-node bifurcation with the appearance of two positive steady states. In this limit case, the curve $\gamma_{1}$ of the function $x_{1} \mapsto F_{1}\left(x_{1}\right)$ is tangent to the curve $\gamma_{2}$ of the function $x_{1} \mapsto F_{2}\left(x_{1}\right)$. Consequently, the equation $F_{1}\left(x_{1}\right)=F_{2}\left(x_{1}\right)$ can have a solution $x_{1}$ on $\left[0, S_{i n}\right]$ which depends on $S_{i n}$ and $D$, where this solution $x_{1}\left(S_{i n}, D\right)$ must satisfy a second equation $F_{1}^{\prime}\left(x_{1}\right)=F_{2}^{\prime}\left(x_{1}\right)$ whose solution can define the curve $\Upsilon_{3}$. Thus, we define the set

$$
\Upsilon_{3}:=\left\{\left(S_{i n}, D\right): F_{1}\left(x_{1}\right)=F_{2}\left(x_{1}\right) \text { and } F_{1}^{\prime}\left(x_{1}\right)=F_{2}^{\prime}\left(x_{1}\right)\right\},
$$


which is a curve in the generic case (see Fig. 4). Table 2 shows the existence and local stability of steady states $\mathcal{E}_{0}, \mathcal{E}_{1}, \mathcal{E}_{1}^{*}$ and $\mathcal{E}_{2}^{*}$ in four regions labeled as $\mathcal{J}_{k}$, $k=0, \ldots, 3$, of the operating diagram shown in Fig. 4. In Table 2, the letter $\mathrm{S}$ (resp. U) means stable (resp. unstable) steady state. Absence of letter means that the corresponding steady state does not exist.

Table 2. Existence and local stability of steady states according to regions in the operating diagram of Fig. 4.

\begin{tabular}{lcccc}
\hline Region & $\mathcal{E}_{0}$ & $\mathcal{E}_{1}$ & $\mathcal{E}_{1}^{*}$ & $\mathcal{E}_{2}^{*}$ \\
\hline$\left(S_{i n}, D\right) \in \mathcal{J}_{0}$ & $\mathrm{~S}$ & & & \\
$\left(S_{i n}, D\right) \in \mathcal{J}_{1}$ & $\mathrm{U}$ & $\mathrm{S}$ & & \\
$\left(S_{i n}, D\right) \in \mathcal{J}_{2}$ & $\mathrm{U}$ & $\mathrm{S}$ & $\mathrm{S}$ & $\mathrm{U}$ \\
$\left(S_{i n}, D\right) \in \mathcal{J}_{3}$ & $\mathrm{U}$ & $\mathrm{U}$ & $\mathrm{S}$ & \\
\hline
\end{tabular}
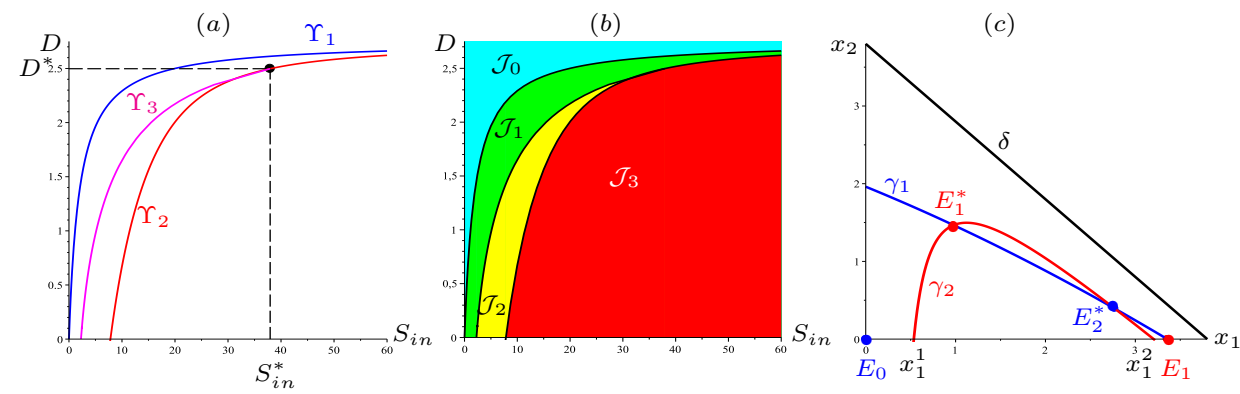

Fig. 4. (a) The curves $\Upsilon_{i}, i=1,2,3$ when $\Upsilon_{2}$ and $\Upsilon_{3}$ intersect in $\left(S_{i n}^{*}, D^{*}\right) \simeq(37.95,2.49)$. (b) The corresponding operating diagram of (1.2). (c) Bistability and multiplicity of positive steady states when $\left(S_{\text {in }}, D\right)=(3.8,0.5) \in \mathcal{J}_{2}$.

Fig. 4 illustrates the operating diagram of (1.2) where the curves $\Upsilon_{i}, i=1,2,3$ separate the plane $\left(S_{i n}, D\right)$ in at most four regions $\mathcal{J}_{k}, k=0, \ldots, 3$. All regions appear in Figs. $4(b)$ and $5(b)$. The region $\mathcal{J}_{3}$ (resp. $\mathcal{J}_{2}$ ) is empty in case $(c)$ in Fig. 5 (resp. $(a)$ ).

The transition from the region $\mathcal{J}_{0}$ to the region $\mathcal{J}_{1}$ by the curve $\Upsilon_{1}$ corresponds to a transcritical bifurcation making the steady state $\mathcal{E}_{0}$ unstable with the appearance of an LES steady state $\mathcal{E}_{1}$. The transition from the region $\mathcal{J}_{1}$ to the region $\mathcal{J}_{3}$ by the curve $\Upsilon_{2}$ corresponds to a transcritical bifurcation making $\mathcal{E}_{1}$ unstable with the appearance of the positive steady state $E^{*}$ which is LES. The transition from the region $\mathcal{J}_{1}$ to the region $\mathcal{J}_{2}$ by the curve $\Upsilon_{3}$ corresponds to a saddle-node bifurcation with the appearance of two positive steady states $\mathcal{E}_{1}^{*}$ which is LES and $\mathcal{E}_{2}^{*}$ which is unstable. The transition from the region $\mathcal{J}_{2}$ to the region $\mathcal{J}_{3}$ by the curve $\Upsilon_{2}$ corresponds to a transcritical bifurcation when the unstable steady state $\mathcal{E}_{2}^{*}$ disappears and $\mathcal{E}_{1}$ becomes unstable. 
(a)

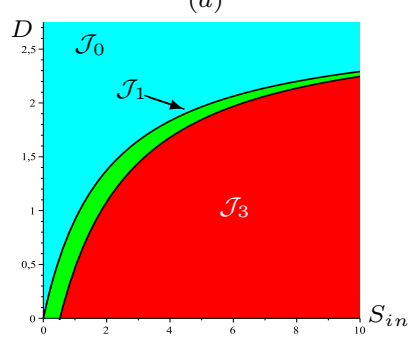

(b)

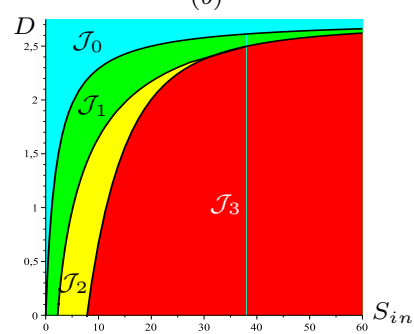

$(c)$

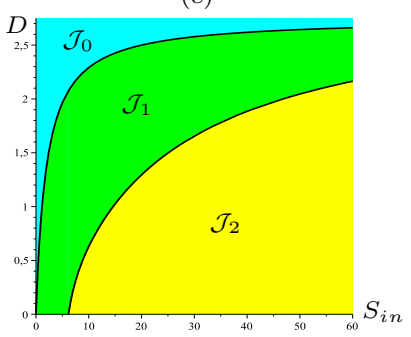

Fig. 5. Effect of variation of the parameter $L_{2}$ on the operating diagram: (a) $L_{2}=0.1$, (b) $L_{2}=1.5$, (c) $L_{2}=10$.

Finally, the operating diagram in Fig. 5 shows the effect of the parameter $L_{2}$ in the growth function (2.1) on the emergence of the bistability region $\mathcal{J}_{2}$ (in yellow). When the parameter $L_{2}$ increases, the operating diagram illustrates how the coexistence region $\mathcal{J}_{3}$ (in red) is reduced and even disappears. To maintain the coexistence of species, the ideal control parameter values of $D$ and $S_{i n}$ should be chosen in $\mathcal{J}_{3}$.

To expose all bifurcations already mentioned and the effect on the concentrations of species at steady state as the control parameter $S_{\text {in }}$ varies while $D=0.5$, we study the one-parameter bifurcation diagram for system (1.2). Fig. 6 shows that the washout steady state $\mathcal{E}_{0}$ loses its stability by a transcritical bifurcation with $\mathcal{E}_{1}$ at the green diamond point for $S_{i n} \simeq 0.44$. Then, a saddle-node bifurcation occurs at cyan circle point for $S_{i n} \simeq 3.29$ between $\mathcal{E}_{1}^{*}$ and $\mathcal{E}_{2}^{*}$. Finally, the steady state $\mathcal{E}_{1}$ loses its stability by a transcritical bifurcation with $\mathcal{E}_{2}^{*}$ which disappears at the green diamond point for $S_{i n} \simeq 9.35$.
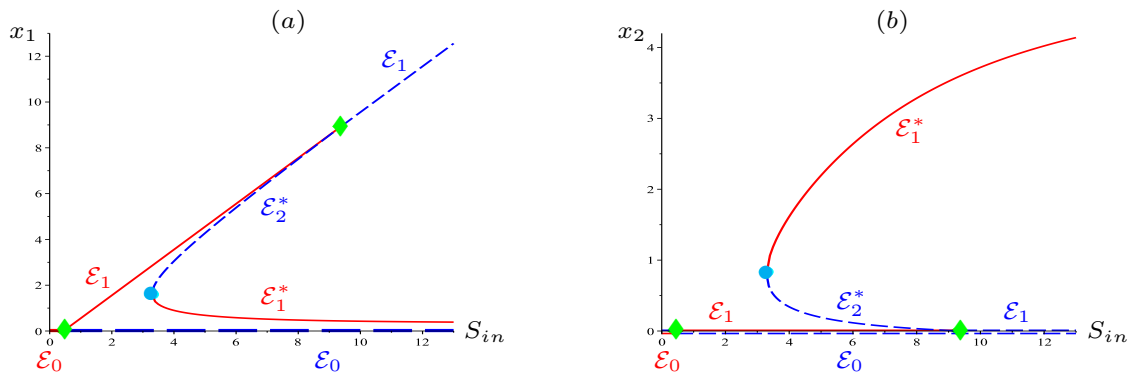

Fig. 6. One-parameter bifurcation diagrams when $D=0.5$ showing the effect on the components $x_{1}$ and $x_{2}$ of all steady states as $S_{i n}$ varies. Blue dashed curves correspond to unstable steady states and red solid curves to LES steady states. The green solid diamonds represent the transcritical bifurcations while the cyan solid circles represent the saddle-node bifurcations.

\section{Numerical simulations}

To validate the results of the previous section, we consider the growth functions given by (2.1) and the set of parameter values provided in Table 3 which were used 
for plotting the operating diagram of Fig. 4. In this section, we plot the solutions of the reduced system (2.4) in the variables $x_{1}$ and $x_{2}$. The phase portraits for system (2.4) illustrate the solutions in the $x_{1}-x_{2}$ plane for several initial conditions.
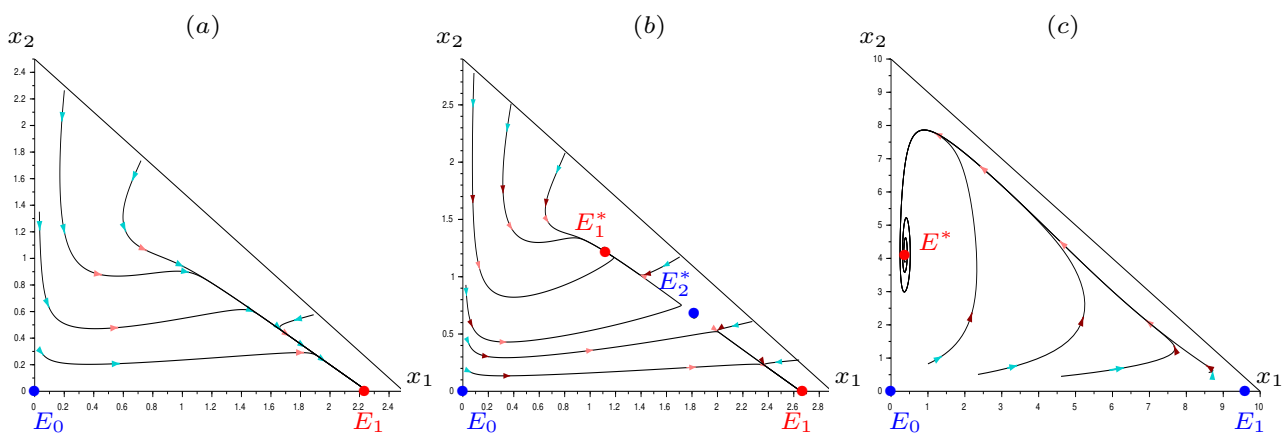

Fig. 7. Phase portraits for (2.4) according to regions in the operating diagram of Fig. 4: (a) Global convergence to $E_{1}$ for $\left(S_{i n}, D\right) \in \mathcal{J}_{1}$. (b) Bistability of $E_{1}^{*}$ and $E_{1}$ for $\left(S_{i n}, D\right) \in \mathcal{J}_{2}$. (c) Global convergence to $E^{*}$ for $\left(S_{i n}, D\right) \in \mathcal{J}_{3}$.

Fig. 7 (a) illustrates the global convergence to $E_{1}$ which is GAS in $M^{o}$ when the system has no positive steady state where $\left(S_{i n}, D\right)=(2.5,0.3) \in \mathcal{J}_{1}$. Fig. $7(b)$ illustrates the multiplicity of positive steady states and the bistability of

$$
E_{1} \simeq(2.655,0) \text { and } E_{1}^{*} \simeq(1.111,1.223)
$$

which are LES while $E_{2}^{*}$ and $E_{0}$ are unstable when $\left(S_{i n}, D\right)=(2.9,0.3)$ is chosen in the bistability region $\mathcal{J}_{2}$. In this case, the numerical simulations show the bistability with two basins of attraction, which are separated by the stable manifold of saddle point $E_{2}^{*}$. One basin attracts the solutions to the positive steady state $E_{1}^{*}$ and the other to the steady state $E_{1}$. Fig. 7 (c) illustrates the case when $\left(S_{i n}, D\right)=$ $(10,0.45) \in \mathcal{J}_{3}$ where there exists a unique positive steady state

$$
E^{*} \simeq(0.381,4.165)
$$

which is LES. Numerical simulations show the global convergence to $E^{*}$ for any positive initial condition.

\section{Conclusion}

In this work, we have analyzed mathematically and through numerical simulations an interspecific density-dependent model of two microbial species in a chemostat competing for a nutrient proposed by El Hajji [6], taking into account the predatorprey relationship. With the same dilution rates, three order model (1.2) can be reduced to second order model (2.4). Thus, we have studied the reduced model (2.4) since the asymptotic behavior of each steady state of (1.2) is derived from the reduced model (2.4). Under general monotonic density-dependent growth functions, we give, in a first step, a complete analysis for the existence and stability of all steady 
states of model (2.4) by using the nullcline method. We have shown the multiplicity of positive steady states, where the number of positive steady states depends on the relative positions of the numbers $\tilde{x}_{1}, x_{1}^{1}$ and $x_{1}^{2}$, namely, three cases of (3.7) must be distinguished. Furthermore, the stability of a positive steady state $E^{*}$ depends on the relative positions of the curves of the nullclines defined by (3.4).

On the other hand, we determined the operating diagram which depicts the asymptotic behavior of the system with respect to control parameters. It shows the occurrence of the bistability region with multiplicity of coexistence steady states that can bifurcate only through saddle-node bifurcations or transcritical bifurcations. To ensure the coexistence between the prey and predator species in the chemostat, the ideal control parameter values of $D$ and $S_{i n}$ should be chosen in the coexistence region.

The increase in the value of $L_{2}$, that is, the decrease in the dynamics of predation reflects on the operating diagram the direct impact of the inhibition of predation on the prey. Indeed, a bistability region emerges while the coexistence region is reduced and then disappears by increasing this inhibition parameter. When only $S_{i n}$ varies, the one-parameter bifurcation diagram illustrates all bifurcations and shows the effect on the concentrations of prey and predator species at steady state. The numerical simulations show that the system may exhibit bistability with convergence either to the coexistence of prey and predator species or to the exclusion of the predator species when the growth functions are given by (2.1).

\section{Acknowledgments}

The first author thanks the Tunisian Ministry of Higher Education, Scientific Research and Technology for financial support and INRAE for hosting her in Montpellier during the preparation of this work. This work was supported by the EuroMediterranean research network TREASURE (http://www.inra.fr/treasure). The authors thank the anonymous reviewers for their constructive comments which have greatly improved this work.

\section{Appendix A. Proofs}

In this Appendix, we give the proofs of all propositions and lemmas.

Proof of Proposition 2.1. From (H0), the vector field defined by (1.2) is continuously differentiable. Hence, the existence and the uniqueness of solution to initial value problems hold. Thanks to (H1), if $S=0$ then $\dot{S}=D S_{\text {in }}>0$. Thus, no trajectory can leave the positive octant $\mathbb{R}_{+}^{3}$ by crossing the boundary face $S=0$. In addition, if $x_{1}=0$ and $x_{2}=0$ then $\dot{x}_{1}=0$ and $\dot{x}_{2}=0$ and the set

$$
\Gamma_{0}=\left\{\left(S, x_{1}, x_{2}\right) \in \mathbb{R}_{+}^{3}: S>0, x_{1}=0, x_{2}=0\right\}
$$

is invariant under system (1.2) because the function

$$
t \longmapsto\left(S(t), x_{1}(t), x_{2}(t)\right)=\left(S_{\text {in }}+\left(S(0)-S_{\text {in }}\right) e^{-D t}, 0,0\right)
$$


is a solution of (1.2). By uniqueness of solutions, the set $\Gamma_{0}$ cannot be reached in finite time by trajectories for which $x_{1}(0)>0$ or $x_{2}(0)>0$. Furthermore, for $i=1,2$, if $x_{i}=0$ then $\dot{x}_{i}=0$ and the set

$$
\Gamma_{i}=\left\{\left(S, x_{1}, x_{2}\right) \in \mathbb{R}_{+}^{3}: S \geq 0, x_{i}=0, x_{j} \geq 0\right\}, \quad j=1,2, i \neq j
$$

is invariant under system (1.2) because the function

$$
t \longmapsto\left(S_{i n}+\left(S(0)-S_{i n}\right) e^{-D t}, 0, x_{2}(0) e^{-D t}\right) \quad\left(\text { resp. } t \longmapsto\left(S(t), x_{1}(t), 0\right)\right)
$$

is a solution of (1.2), thanks to (H4). By uniqueness of solutions, the set $\Gamma_{i}$ cannot be reached in finite time by trajectories for $x_{i}(0)>0$. Therefore, the solutions remain non-negative.

Let $z=S+x_{1}+x_{2}$ be the total mass density. The sum of the three equations of (1.2) yields

$$
\dot{z}=-D\left(z-S_{i n}\right)
$$

then

$$
z(t)=S_{\text {in }}+\left(z(0)-S_{\text {in }}\right) e^{-D t} .
$$

We deduce that

$$
0 \leq z(t)<\max \left(z(0), S_{\text {in }}\right), \quad \text { for all } \quad t \geq 0 .
$$

Therefore, the solutions of (1.2) are positively bounded and are defined for all $t \geq 0$. From (A.1), it can be deduced that the set $\Omega$ is positively invariant and is a global attractor for (1.2).

Proof of Proposition 3.1. If $x_{2}=0$, from the first equation of (3.1), $x_{1}$ is the solution of the equation

$$
f_{1}\left(S_{\text {in }}-x_{1}, 0\right)=D .
$$

From Hypotheses (H0) to (H2) and definition (3.2) of $\lambda$, we have $S_{i n}-x_{1}=\lambda(D)$, that is, $x_{1}=S_{i n}-\lambda(D)$ which is positive if and only if (3.3) holds.

Proof of Lemma 3.2. Let $l_{1}$ be a fixed line defined by $x_{1}=b_{1}$ that intersects the line $\delta$ defined by $x_{1}+x_{2}=S_{\text {in }}$ at point $\left(b_{1}, c_{2}\right)$ where $c_{2}=S_{\text {in }}-b_{1}$ (see Fig. 1(b)). From hypotheses (H0) to (H3), we see that the function $x_{2} \longmapsto f_{1}\left(S_{i n}-b_{1}-x_{2}, x_{2}\right)$ is decreasing from $f_{1}\left(S_{i n}-b_{1}, 0\right)$ for $x_{2}=0$ to 0 for $x_{2}=c_{2}$. Therefore, there exists a unique $b_{2} \in\left[0, c_{2}\right.$ [ such that $f_{1}\left(S_{i n}-b_{1}-b_{2}, b_{2}\right)=D$ if and only if

$$
f_{1}\left(S_{i n}-b_{1}, 0\right) \geq D
$$

which is equivalent to $b_{1} \leq \tilde{x}_{1}$, thanks to (H2) and $\tilde{x}_{1}=S_{i n}-\lambda(D)$ is the unique solution of equation (A.2). Therefore, we have shown that for all $b_{1} \in\left[0, \tilde{x}_{1}\right]$ there exists a unique $b_{2} \in\left[0, c_{2}\right.$ [ such that $f_{1}\left(S_{i n}-b_{1}-b_{2}, b_{2}\right)=D$, that is, each line $l_{1}$ meets the set $f_{1}\left(S_{i n}-x_{1}-x_{2}, x_{2}\right)=D$ exactly once if $0 \leqslant b_{1} \leqslant \tilde{x}_{1}$ and not at all if $b_{1}>\tilde{x}_{1}$. Thus, we define the function $F_{1}$ by $b_{2}=F_{1}\left(b_{1}\right)$. The graph $\gamma_{1}$ of this 
function lies in $M^{o}$ (see Fig. 1(b)). By the implicit function theorem, the function $F_{1}$ is smooth and decreasing. Indeed, using (H2)-(H3), it follows that

$$
-1<F_{1}^{\prime}\left(x_{1}\right)=-\frac{\frac{\partial f_{1}}{\partial x_{1}}\left(x_{1}, F_{1}\left(x_{1}\right)\right)}{\frac{\partial f_{1}}{\partial x_{2}}\left(x_{1}, F_{1}\left(x_{1}\right)\right)}=\frac{\frac{\partial f_{1}}{\partial S}}{-\frac{\partial f_{1}}{\partial S}+\frac{\partial f_{1}}{\partial x_{2}}}<0 .
$$

Furthermore, if $x_{2}=0$ then

$$
F_{1}\left(x_{1}\right)=0 \quad \Leftrightarrow \quad f_{1}\left(S_{i n}-x_{1}, 0\right)=D \quad \Leftrightarrow \quad x_{1}=\tilde{x}_{1}=S_{i n}-\lambda(D),
$$

and if $x_{1}=0$, then

$$
F_{1}(0)=x_{2} \quad \Leftrightarrow \quad f_{1}\left(S_{i n}-x_{2}, x_{2}\right)=D .
$$

From (H0) to (H3), we see that the function

$$
x_{2} \longmapsto f_{1}\left(S_{\text {in }}-x_{2}, x_{2}\right)
$$

is decreasing from $f_{1}\left(S_{i n}, 0\right)$ for $x_{2}=0$ to 0 for $x_{2}=S_{i n}$. Consequently, the equation $f_{1}\left(S_{i n}-x_{2}, x_{2}\right)=D$ has a solution $\left.\tilde{x}_{2} \in\right] 0, S_{i n}$ [ if and only if $f_{1}\left(S_{i n}, 0\right)>D$, that is, condition (3.3) holds. If such an $\tilde{x}_{2}$ exists, then it is unique.

Proof of Lemma 3.3. Let $\phi_{2}$ be the function defined by

$$
\phi_{2}\left(x_{1}\right):=f_{2}\left(S_{i n}-x_{1}, x_{1}\right) .
$$

Under hypothesis (H0)-(H4), $\phi_{2}$ is positive and continuous on $\left[0, S_{i n}\right]$, nonmonotonic and $\phi_{2}(0)=\phi_{2}\left(S_{i n}\right)=0$. Therefore, the equation

$$
f_{2}\left(S_{\text {in }}-x_{1}, x_{1}\right)=D
$$

has a solution in $\left[0, S_{i n}\right]$ if and only if

$$
\max _{x_{1} \in\left[0, S_{i n}\right]} f_{2}\left(S_{i n}-x_{1}, x_{1}\right) \geq D
$$

which is the condition (3.6).

Proof of Lemma 3.4. Assume that hypotheses (H0) to (H5) and condition (3.6) hold. From Lemma 3.3, the equation $f_{2}\left(S_{i n}-x_{1}, x_{1}\right)=D$ has two solutions $x_{1}^{1}$ and $x_{1}^{2}$ in $\left[0, S_{i n}\right]$. Let us prove that $\left(x_{1}, F_{2}\left(x_{1}\right)\right) \in M^{o}$ for all $\left.x_{1} \in\right] x_{1}^{1}, x_{1}^{2}\left[\right.$. Let $l_{1}$ be a fixed line defined by $x_{1}=b_{1}$ that intersects the line $\delta$ defined by $x_{1}+x_{2}=S_{i n}$ at point $\left(b_{1}, c_{2}\right)$ where $c_{2}=S_{i n}-b_{1}$. From hypotheses (H0) to (H2), we see that the function $x_{2} \longmapsto f_{2}\left(S_{i n}-b_{1}-x_{2}, b_{1}\right)$ is decreasing from $f_{2}\left(S_{i n}-b_{1}, b_{1}\right)$ for $x_{2}=0$ to 0 for $x_{2}=c_{2}$. Therefore, there exists a unique $b_{2} \in\left[0, c_{2}[\right.$ such that $f_{2}\left(S_{\text {in }}-b_{1}-b_{2}, b_{1}\right)=D$ if and only if

$$
f_{2}\left(S_{\text {in }}-b_{1}, b_{1}\right) \geq D,
$$

which is equivalent to $b_{1} \in\left[x_{1}^{1}, x_{1}^{2}\right]$, using (H0) to (H5) and condition (3.6) (see Figs. 1(a,c)). Therefore, we have shown that for all $b_{1} \in\left[x_{1}^{1}, x_{1}^{2}\right]$ there exists a unique $b_{2} \in\left[0, c_{2}\right]$ such that $f_{2}\left(S_{i n}-b_{1}-b_{2}, b_{1}\right)=D$. Hence, we define the function 
$F_{2}$ by $b_{2}=F_{2}\left(b_{1}\right)$. The graph $\gamma_{2}$ of this function lies in $M^{o}$. Using the implicit function theorem and assumptions (H2)-(H3), we obtain

$$
-1<F_{2}^{\prime}\left(x_{1}\right)=-\frac{\frac{\partial f_{2}}{\partial x_{1}}\left(x_{1}, F_{2}\left(x_{1}\right)\right)}{\frac{\partial f_{2}}{\partial x_{2}}\left(x_{1}, F_{2}\left(x_{1}\right)\right)}=-1+\frac{\frac{\partial f_{2}}{\partial x_{1}}}{\frac{\partial f_{2}}{\partial S}} .
$$

In addition, when $x_{2}=0$, we have $F_{2}\left(x_{1}\right)=0$ is equivalent to $f_{2}\left(S_{i n}-x_{1}, x_{1}\right)=D$, that is, $x_{1}=x_{1}^{1}$ or $x_{1}=x_{1}^{2}$. This completes the proof.

Proof of Proposition 3.7. At $E_{0}$, the Jacobian matrix of reduced model (2.4) is written as follows:

$$
J_{E_{0}}=\left[\begin{array}{cc}
f_{1}\left(S_{i n}, 0\right)-D & 0 \\
0 & -D
\end{array}\right] .
$$

The eigenvalues are $-D$ and $f_{1}\left(S_{i n}, 0\right)-D$, which are negative if and only if $S_{i n}<$ $\lambda(D)$.

The Jacobian matrix at $E_{1}$ is given by

$$
J_{E_{1}}=\left[\begin{array}{cc}
-\tilde{x}_{1} \frac{\partial f_{1}}{\partial S} & \tilde{x}_{1}\left(-\frac{\partial f_{1}}{\partial S}+\frac{\partial f_{1}}{\partial x_{2}}\right) \\
0 & f_{2}\left(S_{i n}-\tilde{x}_{1}, \tilde{x}_{1}\right)-D
\end{array}\right] .
$$

The eigenvalues are on the diagonal. They are negative if and only if

$$
f_{2}\left(\lambda(D), S_{\text {in }}-\lambda(D)\right)<D,
$$

which is equivalent to Case 1 or Case 3 holds, thanks to (3.8) (see Fig. 1(a)).

The Jacobian matrix at a positive steady state $E^{*}$ is given by

$$
J_{E^{*}}=\left[\begin{array}{cc}
-x_{1}^{*} \frac{\partial f_{1}}{\partial S} & -x_{1}^{*}\left(\frac{\partial f_{1}}{\partial S}+\frac{\partial f_{1}}{\partial x_{2}}\right) \\
-x_{2}^{*}\left(\frac{\partial f_{2}}{\partial S}+\frac{\partial f_{2}}{\partial x_{1}}\right) & -x_{2}^{*} \frac{\partial f_{2}}{\partial S}
\end{array}\right] .
$$

Since

$$
\operatorname{tr} J_{E^{*}}=-\left(x_{1}^{*} \frac{\partial f_{1}}{\partial S}+x_{2}^{*} \frac{\partial f_{2}}{\partial S}\right)<0,
$$

then the real part of the eigenvalues of $J_{E^{*}}$ are negative if and only if

$$
\operatorname{det} J_{E^{*}}=x_{1}^{*} x_{2}^{*}\left(\frac{\partial f_{2}}{\partial S} \frac{\partial f_{1}}{\partial x_{2}}+\frac{\partial f_{2}}{\partial x_{1}} \frac{\partial f_{1}}{\partial S}-\frac{\partial f_{2}}{\partial x_{1}} \frac{\partial f_{1}}{\partial x_{2}}\right)>0 .
$$

Using the derivatives expressions of $F_{1}$ and $F_{2}$, we can write

$$
F_{1}^{\prime}-F_{2}^{\prime}=\frac{\frac{\partial f_{2}}{\partial S} \frac{\partial f_{1}}{\partial x_{2}}+\frac{\partial f_{2}}{\partial x_{1}} \frac{\partial f_{1}}{\partial S}-\frac{\partial f_{2}}{\partial x_{1}} \frac{\partial f_{1}}{\partial x_{2}}}{\frac{\partial f_{2}}{\partial S}\left(\frac{\partial f_{1}}{\partial x_{2}}-\frac{\partial f_{1}}{\partial S}\right)} .
$$

As a consequence, one has

$$
\operatorname{det} J_{E^{*}}=\left(F_{2}^{\prime}-F_{1}^{\prime}\right) x_{1}^{*} x_{2}^{*} \frac{\partial f_{2}}{\partial S}\left(\frac{\partial f_{1}}{\partial S}-\frac{\partial f_{1}}{\partial x_{2}}\right) .
$$

From (H2) and (H3), it follows that

$$
\operatorname{det} J_{E^{*}}>0 \quad \Leftrightarrow \quad F_{2}^{\prime}\left(x_{1}^{*}\right)>F_{1}^{\prime}\left(x_{1}^{*}\right) .
$$


Proof of Proposition 3.8. In order to show that the solutions of (2.4) converge to one of the steady states of the system for all $t \geq 0$, we apply the method used in $[10,17]$. The curves $\gamma_{1}$ and $\gamma_{2}$ separate the set $M^{o}$ in at most four regions as shown in Fig. 3(a). We define the regions as follows:

$$
\begin{array}{llllll}
I: & \dot{x}_{1}<0, & \dot{x}_{2}<0, & I I: & \dot{x}_{1}<0, & \dot{x}_{2}>0, \\
I I I: & \dot{x}_{1}>0, & \dot{x}_{2}<0, & I V: & \dot{x}_{1}>0, & \dot{x}_{2}>0 .
\end{array}
$$

Consider a trajectory $\left(x_{1}(t), x_{2}(t)\right) \in M^{o}$ starting at a positive initial condition $\left(x_{1}(0), x_{2}(0)\right)$. Let us first prove that if $f_{2}\left(\lambda(D), S_{i n}-\lambda(D)\right)>D$, that is, Case 2 holds (see equivalence (3.8)) and (3.9) has a unique solution, then $E^{*}$ is GAS in $M^{o}$. In this case, the curves $\gamma_{1}$ and $\gamma_{2}$ have only one intersection and separate the set $M^{o}$ in four regions $I, I I, I I I$ and $I V$, see Fig. 3(a). Assume first that $\left(x_{1}(0), x_{2}(0)\right) \in I$. If $\left(x_{1}(t), x_{2}(t)\right)$ remains in $I$ for all $t \geq 0$ then $x_{1}(t)$ and $x_{2}(t)$ are positive decreasing functions, with limits

$$
\lim _{t \rightarrow+\infty} x_{1}(t)=x_{1 \infty} \quad \text { and } \quad \lim _{t \rightarrow+\infty} x_{2}(t)=x_{2 \infty} .
$$

Therefore, $\left(x_{1 \infty}, x_{2 \infty}\right)$ is a steady state of (2.4) which belongs to the closure $\bar{I}$ of the set $I$. Since $E^{*}$ is the only steady state in $\bar{I}$, hence

$$
E^{*}=\left(x_{1 \infty}, x_{2 \infty}\right) .
$$

If $\left(x_{1}(t), x_{2}(t)\right)$ leaves the region $I$ and enters in $I I I$. Then $\left(x_{1}(t), x_{2}(t)\right)$ can be remained in $I I I$ for all $t \geq 0$, where $x_{1}(t)$ is increasing and $x_{2}(t)$ is decreasing. Thus, the limits (A.4) exist and $\left(x_{1 \infty}, x_{2 \infty}\right)$ is a steady state of (2.4) which belongs to $\overline{I I I}$. Since $E^{*}$ and $E_{0}$ are the only steady states in $\overline{I I I}$ and since $E_{0}$ attracts only solutions with $x_{1}(0)=0$, we conclude that (A.5) holds. On the other hand, if $\left(x_{1}(t), x_{2}(t)\right)$ leaves the region $I I I$ and enters in $I V$, then also $\left(x_{1}(t), x_{2}(t)\right)$ can be remained in $I V$ for all $t \geq 0$, where $x_{1}(t)$ and $x_{2}(t)$ are increasing. Thus the limits (A.4) exist and $\left(x_{1 \infty}, x_{2 \infty}\right)$ is a steady state of (2.4) which belongs to $\overline{I V}$. Since $E^{*}$ et $E_{1}$ are the only steady states in $\overline{I V}$ and since $E_{1}$ attracts only solutions with $x_{2}(0)=0$, we conclude that (A.5) holds. The solution $\left(x_{1}(t), x_{2}(t)\right)$ can leave the region $I V$ and enters in $I I$. If $\left(x_{1}(t), x_{2}(t)\right)$ remains in the region $I I$ for all $t \geq 0$ then $x_{1}(t)$ is a positive decreasing function and $x_{2}(t)$ is a positive increasing function. Therefore, the limits (A.4) exist and $\left(x_{1 \infty}, x_{2 \infty}\right)$ is a steady state of (2.4) which belongs to $\overline{I I}$. Since $E^{*}$ et $E_{1}$ are the only steady states in $\overline{I I}$ and since $E_{1}$ attracts only solutions with $x_{2}(0)=0$, we conclude that (A.5) holds. Finally, if $\left(x_{1}(t), x_{2}(t)\right)$ leaves the region $I I$ and enters in $I$, then as shown previously it necessarily tends to $E^{*}$ and hence (A.5) holds. Therefore, $E^{*}$ is GAS in $M^{o}$.

Inversely, if $E^{*}$ is GAS in $M^{o}$, then only the Case 2 or Case 3 can be satisfied, using Proposition 3.6. The Case 3 is impossible since in this case we have $E_{1}$ is LES, using Proposition 3.7. Thus, Case 2 holds. If there exist at least two positive steady states, then $E^{*}$ cannot be GAS in $M^{o}$ which is a contradiction. Hence, there exists a unique positive steady state. 
If $f_{2}\left(\lambda(D), S_{i n}-\lambda(D)\right) \leq D$, then either Case 1 or Case 3 holds where the case $\tilde{x}_{1}=x_{1}^{1}$ or $\tilde{x}_{1}=x_{1}^{2}$ can be considered (see equivalence (3.8)). A similar argument to previous case shows that $E_{1}$ is GAS in $M^{o}$ in this case (see Fig. 3(b)). Inversely, if $E_{1}$ is GAS in $M^{o}$, then the positive steady state $E^{*}$ does not exist and $E_{1}$ is locally asymptotically stable. From the Jacobian matrix at $E_{1}$ (see the proof of Proposition 3.7), one has $f_{2}\left(S_{\text {in }}-\tilde{x}_{1}, \tilde{x}_{1}\right) \leq D$.

The last item of the proposition can be treated with the same manner (see Fig. $3(\mathrm{c}))$.

\section{Appendix B. The particular case for growth functions (2.1)}

The following result determines the maximal number of solutions of equation (3.5) in the particular case of growth functions (2.1).

Lemma Appendix B.1. Consider the specific growth functions (2.1) and assume that (3.6) holds. Then, equation (3.5) has at most two solutions $x_{1}^{1}$ and $x_{1}^{2}$ on $\left[0, S_{i n}\right]$.

Proof. When the growth functions are given by (2.1), from definition (A.3) of the function $\phi_{2}$, we have

$$
\phi_{2}^{\prime}\left(x_{1}\right)=\frac{m_{2} x_{1}^{2}\left(L_{2}-K_{2}\right)-2 m_{2} L_{2}\left(K_{2}+S_{i n}\right) x_{1}+m_{2} S_{i n} L_{2}\left(K_{2}+S_{i n}\right)}{\left[\left(K_{2}+S_{i n}-x_{1}\right)\left(L_{2}+x_{1}\right)\right]^{2}} .
$$

Hence,

$\phi_{2}^{\prime}\left(x_{1}\right)=0 \quad \Leftrightarrow \quad m_{2} x_{1}^{2}\left(L_{2}-K_{2}\right)-2 m_{2} L_{2}\left(K_{2}+S_{i n}\right) x_{1}+m_{2} S_{i n} L_{2}\left(K_{2}+S_{i n}\right)=0$

which has at most two solutions on $] 0, S_{i n}$ [ since it is an algebraic equation of degree two in $x_{1}$. Moreover, under (H0)-(H4), the function $\phi_{2}$ is positive and continuous on $\left[0, S_{i n}\right]$ with $\phi_{2}(0)=\phi_{2}\left(S_{i n}\right)=0$. Consequently, $\phi_{2}$ must have a unique positive extremum on ]0, $S_{i n}[$. Therefore, when (3.6) holds, equation (3.5) may have at most two solutions on $\left[0, S_{i n}\right]$ (see Fig. 1(a)).

The following lemma gives the maximal number of extrema of the function $F_{2}(\cdot)$ with specific growth functions (2.1).

Lemma Appendix B.2. For specific growth functions (2.1), the function $x_{1} \longmapsto$ $F_{2}\left(x_{1}\right)$ has a unique positive extremum on $\left[x_{1}^{1}, x_{1}^{2}\right]$.

Proof. From Lemma 3.4, the equation $f_{2}\left(S_{i n}-x_{1}-x_{2}, x_{1}\right)=D$ defines the function $F_{2}(\cdot)$ that can be expressed explicitly by

$$
F_{2}\left(x_{1}\right)=S_{i n}-x_{1}-\frac{D K_{2}\left(L_{2}+x_{1}\right)}{\left(m_{2}-D\right) x_{1}-D L_{2}} .
$$

A straightforward calculation gives the following expression of the derivative of $F_{2}$ :

$$
F_{2}^{\prime}\left(x_{1}\right)=\frac{-\left(m_{2}-D\right)^{2} x_{1}^{2}+2 D L_{2}\left(m_{2}-D\right) x_{1}+D L_{2}\left(K_{2} m_{2}-D L_{2}\right)}{\left[\left(m_{2}-D\right) x_{1}-D L_{2}\right]^{2}} .
$$


Then, the equation $F_{2}^{\prime}\left(x_{1}\right)=0$ has at most two solutions on $] x_{1}^{1}, x_{1}^{2}[$. In other hand, from Lemma 3.4, the function $F_{2}$ is positive and continuous on $\left[x_{1}^{1}, x_{1}^{2}\right]$ and $F_{2}\left(x_{1}^{1}\right)=F_{2}\left(x_{1}^{2}\right)=0$. Therefore, $F_{2}$ must have a unique positive extremum on ]$x_{1}^{1}, x_{1}^{2}[$ (see Fig. $1(\mathrm{c})$ ).

\section{Appendix C. Parameters used in numerical simulations}

All parameter values used in numerical simulations are provided in Table 3.

Table 3. Parameter values used for $(2.4)$ when $f_{1}$ and $f_{2}$ are given by $(2.1)$.

\begin{tabular}{lcccccccc}
\hline Figure & $m_{1}$ & $K_{1}$ & $L_{1}$ & $m_{2}$ & $K_{2}$ & $L_{2}$ & $S_{i n}$ & $D$ \\
\hline Figs. 1 (a,c) & & & & 2.95 & 1.8 & 1.5 & 5 & 0.5 \\
Fig. 1 (b) & 2.75 & 2 & 1.2 & & & & 5 & 0.5 \\
Fig. 2 (a) & 2.5 & 2 & 1 & 6.7 & 1 & 2 & 2 & 1 \\
Fig. 2 (b) & 1.2 & 1.8 & 2.2 & 3 & 1.8 & 1 & 3 & 0.5 \\
Fig. 2 (c) & 2.3 & 1.8 & 2.2 & 2.2 & 1.8 & 1 & 5 & 0.5 \\
Figs. 4(a,b), 5(b) & 2.75 & 2 & 1.2 & 2.95 & 1.8 & 1.5 & variable & variable \\
Fig. 4 (c) & 2.75 & 2 & 1.2 & 2.95 & 1.8 & 1.5 & 3.8 & 0.5 \\
Fig. 5 (a) & 2.75 & 2 & 1.2 & 2.95 & 1.8 & 0.1 & variable & variable \\
Fig. 5 (c) & 2.75 & 2 & 1.2 & 2.95 & 1.8 & 10 & variable & variable \\
Fig. 6 & 2.75 & 2 & 1.2 & 2.95 & 1.8 & 1.5 & variable & 0.5 \\
Fig. 7 (a) & 2.75 & 2 & 1.2 & 2.95 & 1.8 & 1.5 & 2.5 & 0.3 \\
Fig. 7 (b) & 2.75 & 2 & 1.2 & 2.95 & 1.8 & 1.5 & 2.9 & 0.3 \\
Fig. 7 (c) & 2.75 & 2 & 1.2 & 2.95 & 1.8 & 1.5 & 10 & 0.45 \\
\hline
\end{tabular}

\section{References}

[1] N. Abdellatif, R. Fekih-Salem and T. Sari. Competition for a single resource and coexistence of several species in the chemostat, Math. Biosci. Eng., 13 (2016) 631-652.

[2] M. P. Boer, B. W. Kooi and S. A. L. M. Kooijman. Food chain dynamics in the chemostat, Math. Biosci., 150 (1998) 43-62.

[3] F. Borsali and K. Yadi. Contribution to the study of the effect of the interspecificity on a two nutrients competition model, Int. J. Biomath, 8 (2015) 1550008 (17 pages).

[4] G. J. Butler, S. H. Hsu and P. Waltman. Coexistence of competing predators in a chemostat, J. Math. Biol., 17 (1983) 133-151.

[5] P. De Leenheer, D. Angeli and E. D. Sontag. Crowding effects promote coexistence in the chemostat, J. Math. Anal. Appl., 319 (2006) 48-60.

[6] M. El Hajji. How can inter-specific interferences explain coexistence or confirm the competitive exclusion principle in a chemostat?, Int. J. Biomath, 11 (2018) 1850111 (20 pages).

[7] M. El Hajji, J. Harmand, H. Chaker and C. Lobry. Association between competition and obligate mutualism in a chemostat, J. Biol. Dyn., 3 (2009) 635-647. 
[8] M. El Hajji and A. Rapaport. How mutual inhibition confirms competitive exclusion principle, in: M. Hassine, M. Moakher (Eds.), Proceedings of the 5th Conference on Trends in Applied Mathematics in Tunisia, Algeria, Morocco (TAMTAM'11), Centre de Publication Universitaire (2011) 443-449.

[9] R. Fekih-Salem, J. Harmand, C. Lobry, A. Rapaport and T. Sari. Extensions of the chemostat model with flocculation, J. Math. Anal. Appl., 397 (2013) 292-306.

[10] R. Fekih-Salem, C. Lobry and T. Sari. A density-dependent model of competition for one resource in the chemostat, Math. Biosci., 286 (2017) 104-122.

[11] R. Fekih-Salem, A. Rapaport and T. Sari. Emergence of coexistence and limit cycles in the chemostat model with flocculation for a general class of functional responses, Appl. Math. Model., 40 (2016) 7656-7677.

[12] R. Fekih-Salem and T. Sari. Properties of the chemostat model with aggregated biomass and distinct removal rates, SIAM J. Appl. Dyn. Syst., 18 (2019) 481509.

[13] R. Fekih-Salem, T. Sari and N. Abdellatif. Sur un modle de comptition et de coexistence dans le chmostat, ARIMA. J., 14 (2011) 15-30.

[14] B. Haegeman and A. Rapaport. How flocculation can explain coexistence in the chemostat, J. Biol. Dyn., 2 (2008) 1-13.

[15] S. R. Hansen and S. P. Hubbell. Single-nutrient microbial competition : Qualitative agreement between experimental and theoretically forecast outcomes, Science, (1980) 1491-1493.

[16] J. Harmand, C. Lobry, A. Rapaport and T. Sari. The Chemostat: Mathematical Theory of Microorganism Cultures, Chemical Eng. Ser., Chemostat Bioprocesses Set, Vol. 1 (Wiley, New York, 2017).

[17] M. W. Hirsch and S. Smale. Differential Equations, Dynamical Systems, and Linear Algebra, Academic Press, Inc, (1974).

[18] B. W. Kooi and M. P. Boer. Chaotic behaviour of a predator-prey system in the chemostat, Dyn. Contin. Discrete Impulse Syst. Ser. B App. Algorithms, 10 (2003) 259-272.

[19] S. A. L. M. Kooijman and B. W. Kooi. Existence and stability of microbial predator-prey systems, J. Theoret. Biol., 170 (1994) 75-85.

[20] B. Li and Y. Kuang. Simple food chain in a chemostat with distinct removal rates, J. Math. Anal. Appl., 242 (2000) 75-92.

[21] C. Lobry and J. Harmand. A new hypothesis to explain the coexistence of $n$ species in the presence of a single resource, C. R. Biol., 329 (2006) 40-46.

[22] F. Nasrin and S. M. Sohel Rana. Three species food web in a chemostat, Int. J. App. Sci. Eing, 4 (2011) 301-313.

[23] M. Scheffer, S. Rinaldi, J. Huisman and F.J. Weissing. Why plankton communities have no equilibrium: solutions to the paradox, Hydrobiologia, 491 (2003) $9-18$.

[24] J. K. Schmidt, B. König and U. Reichl. Characterization of a three bacte- 
ria mixed culture in a chemostat: evaluation and application of a quantitative Terminal-Restriction Fragment Length Polymorphism (T-RFLP) analysis for absolute and species specific cell enumeration, Biotechnol. Bioeng., 96 (2007) $738-756$.

[25] H. L. Smith and P. Waltman. The Theory of the Chemostat: Dynamics of Microbial Competition, Cambridge University Press, (Cambridge, UK, 1995).

[26] H.R. Thieme. Convergence results and a Poincar-Bendixson trichotomy for asymptotically autonomous differential equations, J. Math. Biol., 30 (1992) 755763.

[27] G. S. K. Wolkowicz and L. Zhiqi. Direct interference on competition in a chemostat, J. Biomath., 13 (1998) 282-291. 